\title{
Dilemmas in Using Phenomenology to Investigate Elementary School Children Learning English as a Second Language
}

\author{
Zihan Shi \\ University of Victoria
}

\section{Author Note}

I gratefully acknowledge many helpful comments and suggestions from Dr. David Blades and two anonymous reviewers.

\begin{abstract}
This paper is intended for doctoral students and other researchers considering using phenomenology as a methodology to investigate the experiences of children learning English as a second language in an elementary classroom setting. I identify six dilemmas or puzzling challenges likely to arise if researchers adopt a phenomenological approach to conducting research. The six dilemmas fall under two categories: fundamental and situational. Fundamental dilemmas include descriptive versus interpretive; objective versus subjective; and participant voice versus researcher voice. The former focus is on a fundamental understanding of phenomenology as a research method while the latter include language and cultural challenges and limitations of the researchers. Situational dilemmas arise from the challenges an investigator may encounter in using an in-depth interview as a research tool with children from different cultural and language backgrounds. I present these dilemmas so that researchers can understand more readily the challenges they may face in exploring the lived experience of these children.
\end{abstract}

Keywords: phenomenology; English Language Learners; lived experience 


\section{Dilemmas in Using Phenomenology to Investigate Elementary School Children Learning English as a Second Language}

The purpose of this paper is to present six dilemmas researchers might encounter if they adopt phenomenology as their approach in conducting studies with children who are learning English as a second language. The six dilemmas fall into two categories: fundamental and situational. Fundamental dilemmas refer to the dilemmas that arise from phenomenology as a research method. These dilemmas include descriptive versus interpretive; objective versus subjective; and speaking for participants versus speaking for the researcher. In addition, researchers may encounter situational dilemmas that are specific to a study conducted with children learning English as a second language. These dilemmas are likely to pose language and cultural challenges to researchers who do not share the same linguistic and cultural experiences with the children.

In this paper, the term English as a second language (ESL) refers to elementary or secondary students whose first language is not English, yet who are learning ESL in a North American setting. Quite often, at home they speak a language other than English with their immigrant parents (Solano-Flores, 2010). A major educational concern arises over how, with their limited English proficiency, these children might benefit more from classroom instruction (Linan-Thompson, Vaughn, Prater, \& Cirino, 2006; O’Day, 2009; Solano-Flores, 2010).

In studying the issue of how to improve the language proficiency of students learning ESL, the choice of research method depends on the precise nature of the research problem. While a variety of qualitative research methods might shed light on children who learn ESL, phenomenology allows researchers to focus on a specific question: What are the experiences of children learning ESL in the elementary classroom? Through phenomenological interviews, the lived experiences of these learners in elementary school classrooms can be understood. Phenomenological interviews, as the method of investigation into the lived experiences of the learners, allow participants to express their experiences, which in turn, allow investigators to uncover the essence of the human experience.

A phenomenological method provides an opportunity for systematic reflection on the learning experiences of children. It is important because a deeper understanding will support students' meaningful learning. Phenomenology provides opportunity for systematic reflection in understanding learners' experiences in the mainstream classroom. In turn, this reflection may provide educators with information on how an effective teacher engages a group of children with classroom activities that facilitate their classroom learning. Phenomenology is a method that can provide the investigator with insight, to "understand the phenomena of education by maintaining a view of pedagogy as an expression of the whole, and a view of the experiential situation as the topos [common theme] of real pedagogic acting” (van Manen, 1990, p. 7).

\section{Phenomenology}

Phenomenology is a term that appears in two contexts: philosophy and methodology. Phenomenology emerges as a philosophical movement concerning how to look at the world. When taken as a methodology, phenomenology provides aspects of how to conduct qualitative research (Dowling, 2007). This paper will focus on an examination of phenomenology as a research methodology. According to Creswell (2007), a phenomenological study "describes the meaning for several individuals of their lived experiences of a concept or a phenomenon” (p. 57). In other words, it focuses on describing a phenomenon that all the participants have experienced, 
such as learning a second language in a school system. The purpose of the phenomenological method is to "reduce individual experiences with a phenomenon to a description of the universal essence" (Creswell, 2007, p. 58). To achieve this goal, a researcher will usually identify a phenomenon for study. For example, Miller (2003) identified children who experienced life with diabetes. She then collected data from the children who experienced this phenomenon; and then, described what all the experiences had in common, which is considered the essence of the experiences of this group of children. This description provides "what they have experienced" and "how they experienced it" (Creswell, 2007, p. 58).

Creswell (2007) identified five major steps in conducting a phenomenological study. First, the investigator will determine if the phenomenological approach suits the research problem. When the research problem is to understand the common experiences of several individuals about a phenomenon, a phenomenological study is appropriate. Second, the researcher will identify a phenomenon that is pertinent to the research question. For example, when the phenomena of learning ESL in a public school environment is identified, the research question is, "What are these ESL learners' learning experiences?" Third, the investigator will usually collect data through phenomenological interviews with participants who have experienced the phenomenon. Often, data collection takes place through in-depth interviews and multiple interviews (Creswell, 2007). Other forms of data, collected through observation or reviews of journals and art, can be included as well (Creswell, 2007). During an interview, an investigator may ask very general questions, such as, "What have you experienced in terms of this phenomenon?" and "What situations have typically influenced or affected your experiences of this phenomenon?” A fourth step occurs during data analysis; researchers begin with highlighting significant statements and subsequently develop clusters of meaning from these statements into themes. These themes, at the final stage of a phenomenological study, will provide a backbone for a description that presents the essence of the phenomenon. The fifth step, as van Manen (1990) suggests, is to present a meaningful phenomenological description that focuses on a vivid example and/or includes an incident in such details as how one's body feels or how things smell. This description would give the reader the feeling of being there.

What distinguishes phenomenological from other qualitative research is that with an emphasis on the subjective point of view (in terms of consciousness and experiences), phenomenology allows the researcher to reflect on the lived experiences of human existence. This reflection is regarded as a thoughtful process, free from theoretical, prejudicial, and suppositional interference (van Manen, 2007). A researcher may employ phenomenology in his or her systematic search to determine the essential properties and structures of consciousness and conscious experience. In addition, an investigator using a phenomenological approach in his or her study may explore the relationship between the individual and the world and may examine how individuals understand the world through consciousness. As McKenna (1982) has noted, "The thesis that consciousness constitutes the world is the thesis that the being-there (Dasein) for us of the world and of anything that is in it is an achievement (Leistung) of consciousness” (p. 6).

A phenomenological inquiry will bring forward all the peculiarities of the object constituted with or without notice or attention (McKenna, 1982). The object is an unnoticed part of the researcher's environment, which is what is implied by being there for me. The concept of being there for me includes not only the focal object, but also the "horizon of what is explicitly before" (McKenna, 1982, p.7), and even, in a more limited sense, what is to come. 
Phenomenology is concerned with "whatever of the world I am conscious of is there for me through my being conscious of it” (McKenna, 1982, p. 7).

Phenomenological approaches have been used in many areas in the educational field (Coleman, 2001; Vespi \&Yewchuk, 1992); however, in the exploration of the ESL's learning experiences, phenomenology has been used primarily with young adult learners (Catina, 2010; Tananuraksakul, 2009). To my knowledge, there seems to be little phenomenological research that has been conducted with children. However, phenomenological interviews revealing student experiences might be used with advantage because a phenomenological inquiry could allow a deep understanding of children's classroom experiences of learning English and of children's use of English to communicate with teachers and peers. Van Manen (2007) argues that phenomenological research aims at creating formative relations between being and acting, between who we are and how we act, and between thoughtfulness and tact. From a phenomenological point of view, the purpose of research is "always to question the way we experience the world, to want to know the world in which we live as human beings" (van Manen, 1990, p. 5).

In viewing the primary classroom experiences of students learning ESL, we can adopt van Manen's (1990) suggestion that researchers turn to an experience that participants have lived. For example, a phenomenological inquiry into the lived experiences of students learning a second language may start with a description of their lives in the classroom, using descriptions of what they said, what they did, and how they interacted with teachers and peers. It may focus on the strategies they apply when they do not understand the language. A phenomenological question could be: What are learning experiences like for these individuals? Researchers may also focus on a variety of possible human experiences. As van Manen (1990) has noted:

A lived experience does not confront me as something perceived or represented; it is not given to me, but the reality of lived experience is there-for-me because I have a reflexive awareness of it, because I possess it immediately as belonging to me in some sense. Only in thought does it become objective. (p. 35)

In summary, the aim of the phenomenological researcher is to describe the essence of lived experience, which gives the reader a feeling of re-living it. In addition, it provides the tools for discovering something meaningful and insightful; "through meditations, conversations, day dreams, inspirations and other interpretive acts, we assign meaning to the phenomena of lived life" (van Manen, 1990, p. 37). The essence of a phenomenon may be revealed "in such a fashion that we are now able to grasp the nature and significance of this experience in a hitherto unseen way” (p. 37).

\section{Dilemmas, Dilemmas}

Although the phenomenological research method has been well articulated (see Boyd, 2001; Caelli, 2001; Creswell, 1994, 2007; van Manen, 1990), few studies have documented the challenges or dilemmas that researchers encounter while using a phenomenological approach (Miller, 2003). They may encounter dilemmas in using phenomenology as a method, especially in investigating the learning experiences of ESL elementary-school-age children. Two types of dilemmas are presented in this paper on using phenomenology as a research method. The dilemmas are categorized as two groups: fundamental and situational. 
Fundamental dilemmas. Fundamental dilemmas refer to the dilemmas that arise from phenomenology as a research method. In turn, these fundamental dilemmas are of several types, which we can call, in brief, (a) descriptive versus interpretive dilemmas, (b) objective versus subjective dilemmas, and (c) dilemmas regarding participant voice versus researcher voice.

Descriptive versus interpretive dilemmas. One of the essential questions arising from a phenomenological inquiry is whether it results in descriptive scientific research or in an interpretive personal inquiry. Researchers may be in a dilemma as to which viewpoint they are going to take in exploring lived experiences.

Phenomenology has evolved into various schools of thought during its development. Husserl (1982) established a basic "descriptive phenomenology” or the descriptive approach to experiences and objects. A major concern in Husserl's transcendental phenomenology is "the question of how knowledge of the world, or of 'transcendent objects,' is possible” (McKenna, 1982, p.2). In Husserl's view, phenomenology is a rigorous and unbiased study that investigates a phenomenon to achieve an essential understanding of human consciousness and experience (as cited in Dowling, 2007). He understood the life-world to be the pre-reflective experience of individuals, without interpretation or cultural context and he emphasized understanding the primeval form of experience, rather than its interpretation. Later interpreters of Husserl emphasized that researchers should refrain from judgment and examine phenomena through a scientific description of the experiences (Dowling, 2007).

In contrast, other phenomenologists maintain an interpretive perspective on phenomenology. Heidegger, for example, argued that the importance of phenomenology lies in interpretation and understanding (as seen in Racher \& Robinson, 2003). According to this view, lived experiences are an interpretive process. In addition, Heidegger argued that presuppositions should not be eliminated or suspended. That is, a person understands the world from within existence rather than from outside. Researchers can interpret a phenomenon only through their own experiences. The present can be understood only through the past, and the past can be understood only through the present (Racher \& Robinson, 2003).

Objective versus subjective dilemmas. Another question regarding phenomenological inquiry is whether the process is an objective or subjective dilemmas. More specifically, can researchers avoid imposing their personal experiences and biases on the description or analysis of the experiences shared by the others? A question arises as to whether they should make explicit their cultural and historical background as well as any possible biases to ensure that readers understand the context of an interpretation.

Some phenomenologists think that phenomenology is an epistemology. They claim that the fundamental source of knowledge is consciousness and that a systematic analysis of phenomena yields a description of experience and its essence (Racher \& Robinson, 2003). Husserl (1982), for example, argued that the experiences of researchers may be bracketed out because phenomenology provides scientific inquiry with a basis for a systematic way of reaching the essence of an experience. The systematic application of bracketing out researchers' experiences is to make sure that phenomenology is a rigorous scientific universal form of knowledge. Researchers can make their perceptions explicit and bracket their presuppositions through a systematic procedure of phenomenological reduction. Furthermore, they need to determine when the bracketing should happen. Giorgi (1983) argued that bracketing should happen in an analysis phase rather than an interview stage of the research. 
More recent theorists suggest that a phenomenological study is a personal inquiry (Racher \& Robinson, 2003). Van Manen (1990), for example, argued that when researchers try to forget or ignore their own experiences, they might find that "the presupposition persistently creeps back into" their reflections (p. 47). Although researchers should reflect upon their prejudices and make these prejudices explicit, researchers' experiences inevitably are reflected in the process of inquiry. Research findings are generated from the interaction between a researcher and participants (Creswell, 2007). As Ajjawi and Higgs (2007) have pointed out, it is impossible to achieve total objectivity because objectivity is situated in a reality constructed by subjective experiences. Moreover, scholars have asked questions regarding (a) when bracketing should happen; (b) to what extent bracketing should be exerted; and (c) how researchers should examine their own prejudices (van Manen, 1990). Researchers inevitably brought with them their own background and presuppositions because any interpretation of a phenomenon is based on researchers' cultural and historical background and interpretations (van Manen, 1990).

Dilemmas regarding participant voice versus researcher voice. Phenomenological inquiry rests on the caring act of the researcher. Van Manen (1990) argued that research is a caring act because the value of the research lies in knowing the essence of a human phenomenon. He maintained that to orient oneself to a phenomenon always implies a particular interest. Binswanger (1963, as cited in van Manen, 1990) argued that the reverse is also true, because we can understand only phenomena about which we care. "One learns to know only what one loves, and the deeper and fuller the knowledge is to be, the more powerful and vivid must be the love, indeed the passion" (p. 6). Because of a caring act, researchers face this dilemma: Do they speak for their participants or for themselves when conducting and reporting phenomenological research? It is important to identify on whose perspective an inquiry will be based to avoid this dilemma.

For example, if I, as a second language speaker with the experience of learning English as a foreign language, were to conduct a study with children learning ESL--because I care about their learning experiences and am interested in working towards their engagement and meaningful learning experiences--I might go through the following research procedures or steps:

1. Identify the phenomenon, which is learning a second language.

2. Interview the participants in the school system.

3. Learn aspects of the participants' life experiences.

4. Transcribe the interviews.

5. Conduct an analysis of data.

During this process, some revealing insights might be gained in using a phenomenological approach. However, these insights would inevitably be integrated with the researcher's background. In a sense, I would speak through my own perspective rather than through the learners' perspectives even though the interviews allow students to talk about what is going on in their lives. Data analysis will be conducted by the researcher. I may then have to ask myself: Do I have the right to speak for someone else, especially when "someone else" refers to children who may not fully realize they are being represented by me? Do I speak for them adequately? The dilemma is: To what extent should the researcher be privileged about the content? This leads to further questions: Whose experience am I exploring? Is it the same experience that these 
learners encountered in their school system? How can the researcher understand their thoughts, feelings, and meaningful actions?

Situational dilemmas. By situational dilemma, I mean dilemmas which arise from the specific situation of working with children who are learning ESL. Situational dilemmas include dilemmas about language differences, cultural differences, and investigator limitations.

Language differences. Gathering information about a learner's lived experience in phenomenological interviews can be problematic because the language of a child (especially his or her English, if he or she is just learning English) is not fully developed. It may be very difficult for researchers to conduct an in-depth interview in English with children having limited language proficiency. Also, researchers may find it difficult to achieve internal validity due to the age and language limitations of the participants. Even if transcripts and analysis are sent back to children for verification, they may not understand the contents of the transcription and report.

To develop a rich description of a phenomenon, researchers tend to choose an information-rich case--those participants who can illuminate the phenomenon--for detailed study (Denzin \& Lincoln, 2000; Patton, 2002). This method of sampling is consistent with interpretive paradigm research (Llewellyn, Sullivan, \& Minichiello, 1999). Gillham (2000) claimed that the overwhelming strength of the face-to-face, one-to-one interview is the richness of the communication. The qualitative interview is an interaction between the interviewer and a participant "in which the interviewer has a general plan of inquiry, but not a specific set of questions that must be asked using particular words and in a particular order" (Baxter \& Babbie, 2004, p. 325). Ideally, the participants do most of the talking. To understand the lived experience of participants, researchers usually adopt in-depth conversations as a method of data collection, which can be the richest sole source of data if done well (Morse, 1994). In fact, for participants with limited English proficiency, it might be difficult for them to provide a rich description of their lived experiences.

In these circumstances, researchers may need to discover the language with which the learners are the most comfortable. If they are comfortable communicating in their first language, researchers may consider hiring research assistants to conduct the interview using the language(s) in which the children are proficient. Hiring experienced interviewers who can speak the children's first language helps to ease the conflicts caused by language differences and to establish trust between the interviewer and the interviewee. Some have suggested that participants are more likely to disclose their experiences to a person who speaks the same language (Bogdan \& Biklen, 2003). Bogdan and Biklen (2003) have also maintained that getting to know each other, building a relationship, and putting the participants at ease are very important factors in interviewing. Rapport and trust are more likely to establish if the researcher has the same background as the participant. Certainly, it would be easier for participants to express themselves fully in response to interview questions. Conducting the interview in the preferred language when learners have not developed a full range of English proficiency is important because participants will more likely understand the interview questions and their purpose. Also, this strategy will allow the participants to tell their personal stories and lived experiences more fully (Bogdan \& Biklen, 2003). However, a situation in which participants are of diverse linguistic backgrounds, the effect of such a strategy may be seriously restricted due to the demand for hiring multi-lingual interviewers to conduct the interviews in different languages. 
Another suggestion for working with children learning ESL is to prepare prompt questions, questions that help them tell their story. Miller (2003) maintained that starting with the topic of a normal day seems particularly helpful in getting children to tell their stories. Although prompt questions may help them focus on specific events or situations, as Miller (2003) cautioned, they should be open-ended so as to make sure that children can develop a conversation. This idea is in line with van Manen's (1990) view that it is acceptable to use appropriate techniques in a particular study, so long as they are consistent with the general orientation of the methodology. Furthermore, according to Miller (2003), conversations with parents and field notes of the visits will provide supplementary insight into the context of interviewee's lives (Miller, 2003).

Cultural differences. The cultural background of a researcher plays an important role in phenomenological inquiries. Cultural differences may influence how children answer interview questions. Hofstede (1997) has named the following four dimensions of culture: power distance, collectivism versus individualism, femininity versus masculinity, and uncertainty avoidance (from weak to strong). The power distance of a culture refers to how less powerful members in a society accept the fact that power is unevenly distributed. Cultures also differ in the extent to which individuals consider themselves a member of a group and behave accordingly, defining an individualism versus collectivism range. Masculine cultures value competitiveness, assertiveness, ambition, and the accumulation of wealth and material possessions, whereas feminine cultures place more value on relationships and quality of life. Uncertainty avoidance is a cultural quality that refers to the extent to which individuals of the culture attempt to minimize uncertainty.

Children with cultural backgrounds different from that of the host country might answer the adult researcher's questions in a culturally influenced way. Their perception of the authority of the researcher in an interview situation may influence the way how the learners tell their stories and what experiences are revealed. The children may choose to tell the stories in the way they think the researcher wants, instead of describing their real experiences. For example, participants from collective cultures may try to maintain harmony, which may result in avoidance of confrontation and conflict. They might not describe their experiences of conflict with other people because they feel embarrassed. Just as it is important for investigators to understand the cultural background of their participants, it is equally important that researchers learn not to stereotype their participants.

Researcher limitations. Due to language and cultural considerations, researchers might feel limited in conducting research by using a phenomenological inquiry. When conducting quantitative research, researchers can hire research assistants, who share the same linguistic and cultural backgrounds as the participants, to administer surveys, which can be conducted in the participants' native language. After the data collection, research assistants can enter the data. Then, researchers can use statistical analysis to analyze the data and report the results of the study.

In qualitative research, although researchers may conduct a trial interview to see how the interview questions solicit answers, every participant is different (noted especially when working with children). It is even more difficult if these children have not developed language proficiency. On the researchers' part, understanding language and cultural implications takes time to develop. Miller (2003) cautioned against the danger of changing the meaning of data by the addition of adult interpretation. Instead, she suggested using the children's language whenever possible, which makes a member check with children reliable. 
Another way of conducting research is to interview adult or adolescent second language speakers who have gone through the process of learning a second language in childhood. Investigators can use reflections and memories to identify their experience while learning English as a second or additional language. However, the problem now may arise with the use of memories: To what extent does an adult or adolescent interpret learning experiences that happened many years ago? Clearly, various uncertainties complicate phenomenological research methodology when adopted for investigating the lived experiences of children.

Parahoo (1997) has argued that phenomenology stresses that only those who experience phenomena are capable of communicating them to others; therefore, to understand children's experiences of learning ESL, their views and voices cannot be eliminated from the research. Thus, research methods aim to borrow the experience of others to provide a description and interpretation of that experience (van Manen, 1990). In working with children, Miller (2003) admitted that data analysis and the desire to be true to children's perceptions of their situation were perhaps the biggest challenges.

\section{Summary}

Using phenomenology to explore the lived experiences of children learning English as a second language suggests predictable dilemmas demanding examination. This article provides researchers with some insights into the challenges that they might encounter when using this method to work with children from different linguistic and cultural backgrounds. The first three dilemmas, which are descriptive versus interpretive; objective versus subjective and participant voice versus researcher voice, focus on a fundamental understanding of phenomenology, followed by three challenges of working with children from different cultural and linguistic backgrounds using the in-depth interview as a research tool. I have provided suggestions to deflate some of these dilemmas; for others, I have presented flags to alert researchers. Ultimately, when researchers consider phenomenology as a research method, they need to weigh its benefits and limitations. For example, they need to decide how they are going to work with children from different cultural and linguistic backgrounds. An awareness of the possible dilemmas will help researchers decide how to use phenomenology as a research inquiry into the lived experiences. 


\section{References}

Ajjawi, R., \& Higgs, J. (2007). Using hermeneutic phenomenology to investigate how experienced practitioners learn to communicate clinical reasoning. The Qualitative Report 12(4), 612-638.

Baxter, L. A., \& Babbie, E. (2004). The basics of communication research. Belmont, CA: Wadsworth.

Binswanger, L. (1963). Being in the world. London: Souvenir Press.

Bogdan, R. C., \& Biklen, S. K. (2003). Qualitative research for education: An introduction to theories and methods (4th ed.). Upper Saddle River, NJ: Pearson Education Group.

Boyd, C. O. (2001). Phenomenology: The method. In P. L. Munhall (Ed.), Nursing research: A qualitative perspective ( $3^{\text {rd }}$ ed., pp. 93-122). Sudbury, MA: Jones and Bartlett.

Caelli, K. (2001). Engaging with phenomenology: Is it more of a challenge than it needs to be? Quantitative Health Research, 11(2), 273-282.

Catina, K. L. (2010). Outcomes of a sheltered collaborative teaching model for English language learners. Unpublished doctoral dissertation, the Virginia Polytechnic Institute and State University.

Coleman, L. (2001). A “rag quilt:” Social relationships among students in a special high school. Gifted Child Quarterly, 45(3), 164-173.

Creswell, J. W. (1994). Research design: Qualitative and quantitative approaches. Thousand Oaks, CA: Sage.

Creswell, J. W. (2007). Qualitative inquiry and research design: Choosing among five traditions. Thousand Oaks, CA: Sage.

Denzin, N. K., \& Lincoln, Y. S. (2000). Instruction: The discipline and practice of qualitative research. In N. K. Denzin \& Y. S. Lincoln (Eds.), Handbook of qualitative research $\left(2^{\text {nd }}\right.$ ed., pp. 1-29). Thousand Oaks, CA: Sage.

Dowling, M. (2007). From Husserl to van Manen: A review of different phenomenological approaches. International Journal of Nursing Studies, 44(1), 131-142.

Gillham, B. (2000). Case study research methods. London: Continuum.

Giorgi, A. (1983). Concerning the possibility of phenomenological psychological research. Journal of Phenomenological Psychology, 14(2), 129-169.

Hofstede, G. (1997). Cultures and organizations: Software of the mind. New York, NY: McGraw- Hill.

Husserl, E. (1982). Ideas pertaining to a pure phenomenology and to a phenomenological philosophy: General introduction to a pure phenomenology. The Hague: Martinus Nijhoff.

Linan-Thompson, S., Vaughn, S., Prater, K., \& Cirino, P. T. (2006). The response to intervention of English language learners at risk for reading problems. Journal of Learning Disabilities, 39(5), 390-398.

Llewellyn, G., Sullivan, G., \& Minichiello, V. (1999). Designing health research: Sampling in qualitative research. In V. Minichiello, G. Sullivan, K. Greenwood, \& R. Axford (Eds.), 
Handbook for research methods in health sciences (pp. 174-199). Sydney, Australia: Addison-Wesley.

McKenna, W. R. (1982). Husserl's “Introductions to Phenomenology:" Interpretation and critique. The Hague: Martinus Nijhoff.

Miller, S. (2003). Analysis of phenomenological data generated with children as research participants. Nurse Research, 10(4), 68-82.

Morse, J. M. (1994). Critical issues in qualitative research methods. Thousand Oaks, CA: Sage.

O’Day, J. (2009). Good instruction is good for everyone-or is it? English language learners in a balanced literacy approach. Journal of Education for Students Placed at Risk (JESPAR), 14(1), 97-119.

Parahoo, K. (1997). Nursing research: Principles, process and issues. London: Macmillan Press.

Patton, M. Q. (2002). Qualitative research and evaluation methods. Thousand Oaks, CA: Sage.

Racher, F., \& Robinson, S. (2003). Are phenomenology and postpositivism strange bedfellows? Western Journal of Nursing Research, 25(5), 464-481.

Short, D., \& Fitzsimmons, S. (2007). Double the work: Challenges and solutions to acquiring language and academic literacy for adolescent English language learners. Alliance for Excellent Education. Retrieved from http://carnegie.org/publications/searchpublications/pub/207/

Solano-Flores, G. (2010). English language learners. In C. S. Clauss-Ehlers (Ed.), Encyclopedia of cross-cultural school psychology. Springer Science-Business Media.

Tananuraksakul, N. (2009). A phenomenological study of international postgraduate students' feelings of security in an Australian context. Retrieved from http://proceedings.com.au/isana2009/PDF/paper_Tananuraksakul.pdf

Van Manen, M. (1990). Research lived experience: Human science for an action sensitive pedagogy. London, ON: The Althouse Press.

Van Manen, M. (2007). Phenomenology of practice. Phenomenology and Practice, 1(1), 11-30.

Vespi, L., \& Yewchuk, C. (1992). A phenomenological study of the social/emotional characteristics of gifted learning disabled children. Journal for the Education of the Gifted, 16(1), 55-72. 